\title{
Maximum Principle of Discrete Stochastic Control System Driven by Both Fractional Noise and White Noise
}

\author{
Yuecai Han (i) and Zheng Li (i) \\ School of Mathematics, Jilin University, Changchun 130012, China \\ Correspondence should be addressed to Zheng Li; lizheng17@mails.jlu.edu.cn
}

Received 12 April 2020; Accepted 18 May 2020; Published 5 June 2020

Academic Editor: Antonio Iannizzotto

Copyright ( 92020 Yuecai Han and Zheng Li. This is an open access article distributed under the Creative Commons Attribution License, which permits unrestricted use, distribution, and reproduction in any medium, provided the original work is properly cited.

In this paper, we investigate the necessary optimality conditions of the discrete stochastic optimal control problems driven by both fractional noise and white noise. Here, the admissible control region is not necessarily convex. The corresponding variational inequalities are obtained by applying the classical variation method and Malliavin calculus. We also apply the stochastic maximum principle to a linear-quadratic optimal control problem to illustrate the main result.

\section{Introduction}

We consider a stochastic control problem for state process driven by both general white noise and fractional noise with
Hurst parameter $H \in((1 / 2), 1)$. More precisely, the state of the system is described as the following stochastic difference equation:

$$
\left\{\begin{array}{l}
x_{t_{k+1}}=f\left(x_{t_{k}}, v_{t_{k}}\right)+b\left(x_{t_{k}}, v_{t_{k}}\right) g_{t_{k}}+\sigma\left(x_{t_{k}}, v_{t_{k}}\right) \omega_{t_{k}}, \quad k=0,1, \ldots, N-1, \\
x_{t_{0}}=x_{0} \in \mathbb{R}^{n}
\end{array}\right.
$$

where the functions $f, b$, and $\sigma$ and control variables $u_{t_{k}}$ are introduced in Section 2. The cost functional is defined as follows:

$$
J(v)=E\left[\sum_{k=0}^{N-1} l\left(x_{t_{k}}, v_{t_{k}}\right)+h\left(x_{t_{N}}\right)\right]
$$

where the functions $l$ and $h$ are also introduced in Section 2.

Optimal control problems have a variety of applications in fields such as engineering, financial mathematics, and physics. The maximum principle is one of the main contents of modern control theory. As a necessary condition of the deterministic optimal control, it was formulated by Pontryagin and his group [1]. It states that any optimal control along with the optimal state trajectory must solve a
Hamiltonian system, which is a two-point boundary value problem, plus a maximum condition of Hamiltonian. The theory was then developed extensively, and different versions of the maximum principle were derived.

With the development of the optimal control theory, some researchers began to work on the discrete case by following the Pontryagin maximum principle for continuous optimal control problems. However, the fact has been verified that the discrete case was unlike the continuous case. By imposing convexity requirement, some researchers [2, 3] gave a derivation of the discrete maximum principle. A discrete optimization problem without assumptions of convexity and smoothness was shown by Mardanov et al. [4]. Taking into account the specific character of the discrete system, they obtained a necessary optimality condition 
which is not formulated in terms of the Hamilton-Pontryagin function.

Naturally, with the emergence of stochastic problems, more and more researchers extend the maximum principle to the stochastic case. Kushner [5] employed the spike variation and Neustadt's variational principle [6] to derive a stochastic maximum principle. Based on Girsanov transformations, Haussmann [7] extensively investigated the necessary conditions of stochastic optimal state feedback controls for systems with nondegenerate diffusion coefficients. Bismut [8] derived the adjoint equation via the martingale representation theorem. Peng [9] first considered the second-order term in the "Taylor expansion" of the variation and obtained a stochastic maximum principle for systems that are possibly degenerate, with control-dependent diffusions and not necessarily convex regions. The form of his maximum principle is quite different from the deterministic ones and reflects the stochastic nature of the problem. With the development of the fractional calculus, Han et al. [10] obtained a maximum principle for the stochastic control problem of general controlled stochastic differential systems driven by fractional Brownian motions (of Hurst parameter $H>(1 / 2)$ ), and the maximum principle involves Malliavin derivatives.

However, as far as the discrete stochastic system, some results for the maximum principle are analogous to the deterministic systems, which are based on the Lagrange multiplier method [11]. Recently, Lin and Zhang [12] developed a maximum principle for optimal control of discrete-time stochastic systems, and the admissible control region was nonconvex. It can be found that, up to now, the existing results appearing on the stochastic discrete version mostly study the systems with general multiplicative noise. Inspiring from this, we study the maximum principle of discrete stochastic systems driven by both fractional noise and white noise by using the classical variational method and Malliavin calculus. The admissible control region is nonconvex.

The rest of this paper is organized as follows. In Section 2 , we introduce some preliminaries and main assumptions needed to study the discrete stochastic control problem driven by both fractional noise and white noise. In Section 3, we derive the necessary conditions that the optimal control should satisfy. In Section 4, an example is given to illustrate the main results. In Section 5, we summarize the methods used and the results obtained.

\section{Preliminaries}

Let $W(t)=\left(W_{1}(t), W_{2}(t), \ldots, W_{d}(t)\right)^{T}, 0 \leq t \leq T$, be a $d$-dimensional standard Brownian motion. Let

$$
\begin{aligned}
Z_{H}(t, s)= & \kappa_{H}\left[\left(\frac{t}{s}\right)^{H-(1 / 2)}(t-s)^{H-(1 / 2)}-\left(H-\frac{1}{2}\right) s^{(1 / 2)-H}\right. \\
& \left.\cdot \int_{s}^{t} u^{H-(3 / 2)}(u-s)^{H-(1 / 2)} \mathrm{d} u\right]
\end{aligned}
$$

with

$$
\kappa_{H}=\sqrt{\frac{2 H((3 / 2)-H)}{\Gamma(H+(1 / 2)) \Gamma(2-2 H)}},
$$

and define

$$
B_{j}^{H}(t)=\int_{0}^{t} Z_{H}(t, s) \mathrm{d} W_{j}(s), \quad 0 \leq t \leq \infty .
$$

Then, $\quad B^{H}(t)=\left(B_{1}^{H}(t), \ldots, B_{d}^{H}(t)\right)^{T}, \quad 0 \leq t \leq T, \quad$ is $\quad$ a $d$-dimensional fractional Brownian motion.

Recall the operators $B_{H, T}^{*}$ (see Eq. (5.35) of $\mathrm{Hu}[13]$ ):

$$
B_{H, T}^{*} f(t)=-\frac{2 H \kappa_{1}}{\kappa_{H}} t^{(1 / 2)-H} u^{H-(1 / 2)} f(u) \mathrm{d} u, \quad 0 \leq t \leq T,
$$

where

$$
\kappa_{1}=\frac{1}{2 H \Gamma(H-(1 / 2)) \Gamma((3 / 2)-H)} .
$$

Let $\xi_{1}, \xi_{2}, \ldots, \xi_{k}, \ldots$ be an orthonormal basis of $L^{2}([0, T])$ such that $\xi_{k}, k=1,2, \ldots$, are smooth functions on $[0, T]$. Let $p$ be the set of all polynomials of the standard Brownian motions $W$ over interval $[0, T]$, namely, $p$ contains all elements of the form

$$
F(\omega)=f\left(\int_{0}^{T} \xi_{1}(t) \mathrm{d} B_{t}, \ldots, \int_{0}^{T} \xi_{n}(t) \mathrm{d} B_{t}\right),
$$

where $f$ is a polynomial of $n$ variables. If $F$ is of the above form, then its Malliavin derivative $D_{s} F$ is defined as

$$
D_{s} F=\sum_{i=1}^{n} \frac{\partial f}{\partial x_{i}}\left(\int_{0}^{T} \xi_{1}(t) \mathrm{d} B_{t}, \ldots, \int_{0}^{T} \xi_{n}(t) \mathrm{d} B_{t}\right) \xi_{i}(s), \quad 0 \leq s \leq T .
$$

For any $F \in \mathcal{R}$, we denote the following norm:

$$
\|F\|_{1, p}:=\|F\|_{p}+\sum_{l=1}^{d}\left[E\left(\int_{0}^{T}\left|D_{t}^{l} F\right|^{2} \mathrm{~d} t\right)^{p / 2}\right]^{1 / p}
$$

Let $\mathbb{D}_{1, p}$ denote the Banach space obtained by completing $p$ under the norm $\|\cdot\|_{1, p}$.

Let $\eta_{1}, \eta_{2}, \ldots, \eta_{k}, \ldots$ be an orthonormal basis of $L^{2}([0, T])$ such that $\eta_{k}, k=1,2, \ldots$, are smooth functions on $[0, T]$. Let $p^{H}$ be the set of all polynomials of the fractional Brownian motions $W$ over interval $[0, T]$, namely, $p^{H}$ contains all elements of the form

$$
G(\omega)=g\left(\int_{0}^{T} \eta_{1}(t) \mathrm{d} B_{t}^{H}, \ldots, \int_{0}^{T} \eta_{n}(t) \mathrm{d} B_{t}^{H}\right)
$$

where $g$ is a polynomial of $n$ variables. We define its Malliavin derivative by

$$
D_{s}^{H} F=\sum_{j=1}^{n} \frac{\partial g}{\partial x_{i}}\left(\int_{0}^{T} \eta_{1}(t) \mathrm{d} B_{t}^{H}, \ldots, \int_{0}^{T} \eta_{n}(t) \mathrm{d} B_{t}^{H}\right) \eta_{j}(s),
$$

$$
0 \leq s \leq T \text {. }
$$


Similarly, we define $\|\cdot\|_{H, 1, p}$ and $\mathbb{D}_{H, 1, p}$.

The following duality formula will be used later to solve stochastic optimal control problems (15), (16), and (19).

Lemma 1 (Theorem 6.23 of [13]). Let $f:[0, T] \otimes$ $\left(\Omega, \mathscr{F}, P^{H}\right) \longrightarrow \mathbb{R}$ be jointly measurable, and let $G \in \mathbb{D}_{H, 1,2}$. Then,

$$
E\left[\int_{0}^{T} f(t) \mathrm{d} B_{t}^{H} G\right]=\int_{0}^{T} E\left(f(t) D_{t}^{H} G\right) \mathrm{d} t .
$$

Let $(\Omega, \mathscr{F}, P)$ be a given complete probability space. Let $\left\{\mathscr{F}_{k}\right\}_{k=0}^{N} \subset \mathscr{F}$ be the $\sigma$-field generated by $W_{t_{0}}, W_{t_{1}}, \ldots, W_{t_{N-1}}$ and $\mathscr{F}_{-1}=\{\varnothing, \Omega\}$, where $\left\{W_{t_{k}}\right\}_{k=0,1,2, \ldots, N-1}$ is a sequence of the $d$-dimension Brownian motion.

Similarly, let $\left\{B_{t_{k}}^{H}\right\}_{k=0,1,2, \cdots, N-1}$ be a sequence of the $d$-dimension fractional Brownian motion that satisfies the following conditions, where $(1 / 2)<H<1$ : (i) $\left\{B_{t_{k}}^{H}\right\}_{k=0,1,2, \ldots, N}$ is $\mathscr{F}_{k-1}$-measurable

(ii) The increments of $B_{t_{k}}^{H}$ are stationary and need not be independent

(iii) For every $B_{t_{k}}^{H}=\left(B_{t_{k}}^{H, 1}, B_{t_{k}}^{H, 2}, \ldots, B_{t_{k}}^{H, d}\right)^{\top}, B_{t_{k}}^{H, 1}$, $B_{t_{k}}^{H, 2}, \ldots, B_{t_{k}}^{H, d}$ are independent $\mathbb{R}$-valued Gaussian random variables

(iv) $E\left[B_{t_{k}}^{H}\right]=0$,

$$
\begin{aligned}
& E\left[B_{t_{i}}^{H, l} B_{t_{j}}^{H, l}\right]=(1 / 2)\left(t_{i}^{2 H}+t_{j}^{2 H}-\left|t_{i}-t_{j}\right|^{2 H}\right), \text { where } \\
& l=1,2, \cdots, d
\end{aligned}
$$

Let $\omega_{t_{k}}=W_{t_{k+1}}-W_{t_{k}}$ be the white noise and $g_{t_{k}}=B_{t_{k+1}}^{H}-$ $B_{t_{k}}^{H}$ be the fractional noise.

Let $D$ be a bounded domain, and the space of admissible controls is defined as

$$
U_{\mathrm{ad}}=\left\{U_{k}\right\}_{k=0}^{N-1} \triangleq\left\{v_{t_{k}} \in U_{k} \in \mathbb{R}^{n}: \Omega \longrightarrow D \mid v_{t_{k}} \text { is } \mathscr{F}_{k-1}-\text { measurable and } E\left[v_{t_{k}}^{\top} v_{t_{k}}\right]<+\infty, k=0,1, \ldots N-1\right\}
$$

For the arbitrary bounded random variable $v$ and sufficient small $\varepsilon>0$, we define $\bar{v}=u_{t_{m}}^{*}+\varepsilon v$ for some $m \in\{1,2, \ldots, N-1\}$. Let the admissible control $u_{t_{k}}^{\varepsilon}=\left(1-\delta_{k m}\right) u_{t_{k}}^{*}+\delta_{k m} \bar{v}$, and we can rewrite $u_{t_{k}}^{\varepsilon}$ as $u_{t_{k}}^{\varepsilon}=u_{t_{k}}^{*}+\delta_{k m} \varepsilon v$, where $\delta_{k m}$ is the Kronecker delta, i.e., $\delta_{k m}=1$ when $k=m$ and $\delta_{k m}=0$ when $k \neq m$.

The controlled stochastic system is described as the following discrete stochastic difference equation:

$$
\left\{\begin{array}{l}
x_{t_{k+1}}=f\left(x_{t_{k}}, v_{t_{k}}\right)+b\left(x_{t_{k}}, v_{t_{k}}\right) g_{t_{k}}+\sigma\left(x_{t_{k}}, v_{t_{k}}\right) \omega_{t_{k}}, \quad k=0,1, \ldots, N-1, \\
x_{t_{0}}=x_{0} \in \mathbb{R}^{n}
\end{array}\right.
$$

The cost functional is

$$
J(v)=E\left[\sum_{k=0}^{N-1} l\left(x_{t_{k}}, v_{t_{k}}\right)+h\left(x_{t_{N}}\right)\right] .
$$

Assume that $f, b, \sigma, l$, and $h$ satisfy the following conditions:

$$
\sup _{x \in \mathbb{R}^{n}, u \in \mathbb{R}^{\nu}}\left[\left|f_{x}\left(x_{t_{k}}, u_{t_{k}}\right)\right|+\left|f_{u}\left(x_{t_{k}}, u_{t_{k}}\right)\right|+\sum_{j=1}^{s}\left|b_{x}^{j}\left(x_{t_{k}}, u_{t_{k}}\right)\right|+\sum_{j=1}^{s}\left|b_{u}^{j}\left(x_{t_{k}}, u_{t_{k}}\right)\right|+\sum_{j=1}^{d}\left|\sigma_{x}^{j}\left(x_{t_{k}}, u_{t_{k}}\right)\right|+\sum_{j=1}^{d}\left|\sigma_{u}^{j}\left(x_{t_{k}}, u_{t_{k}}\right)\right|\right] \leq C .
$$

(H1) The function $f: \mathbb{R}^{n} \times \mathbb{R}^{n} \longrightarrow \mathbb{R}^{n}, b=\left(b^{1}, b^{2}\right.$, $\left.\ldots, b^{d}\right): \mathbb{R}^{n} \times \mathbb{R}^{n} \longrightarrow \mathbb{R}^{d}$ and $\sigma=\left(\sigma^{1}, \sigma^{2}, \ldots, \sigma^{d}\right): \mathbb{R}^{n}$ $\times \mathbb{R}^{n} \longrightarrow \mathbb{R}^{d}$ are continuous and differentiable with respect to variables and $u$.

(H2) Assume that there exists a constant $C>0$ such that
(H3) The function $l: \mathbb{R}^{n} \times \mathbb{R}^{n} \longrightarrow \mathbb{R}$ and $h: \mathbb{R}^{n} \longrightarrow$ $\mathbb{R}$ are continuous and differentiable with bounded derivatives.

According to equation (15) and the definition of admissible controls, $u$ and $x \in \mathbb{R}^{n}$. Here, all derivations are for vectors. We have 


$$
\begin{aligned}
& f_{x}=\frac{\partial f(x, u)}{\partial x}, \\
& f_{u}=\frac{\partial f(x, u)}{\partial u}, \\
& b_{x}=\frac{\partial b(x, u)}{\partial x}, \\
& b_{u}=\frac{\partial b(x, u)}{\partial u}, \\
& \sigma_{x}=\frac{\partial \sigma(x, u)}{\partial x} \\
& \sigma_{u}=\frac{\partial \sigma(x, u)}{\partial u}
\end{aligned}
$$

where $f_{x}, f_{u} \in R^{n \times n}$ and $b_{x}, \sigma_{x}, b_{u}, \sigma_{u} \in \mathbb{R}^{n \times d}$.

Our stochastic optimal control problem is to minimize the cost functional $J(v)$ over, namely, to find the optimal $u^{*} \in U_{\text {ad }}$ satisfying

$$
J\left(u^{*}\right)=\inf _{u^{\varepsilon} \in U_{\mathrm{ad}}} J\left(u^{\varepsilon}\right) .
$$

\section{The Maximum Principle}

We have the following theorem as the main result of this paper.

Theorem 1. Let the assumptions $(H 1-H 3)$ hold. If $\left(x_{t_{k}}^{*}, u_{t_{k}}^{*}\right)$ is a solution to optimal control problems (15), (16), and (19) and satisfies the following equation,

$$
\left\{\begin{array}{l}
\phi_{t_{k+1}}=\phi_{t_{k}} f_{x}\left(x_{t_{k}}^{*}, u_{t_{k}}^{*}\right)+\phi_{t_{k}} b_{x}\left(x_{t_{k}}^{*}, u_{t_{k}}^{*}\right) g_{t_{k}}+\phi_{t_{k}} \sigma_{x}\left(x_{t_{k}}^{*}, u_{t_{k}}^{*}\right) \omega_{t_{k}}, \\
\phi_{t_{0}}=I_{n \times n}
\end{array}\right.
$$

where $f_{x}, b_{x}$, and $\sigma_{x}$ are defined above, then there exists the following general maximum principle:

$$
\begin{aligned}
& l_{x}\left(x_{t_{m}}^{*}, u_{t_{m}}^{*}\right) \phi_{t_{m}} \sum_{n=0}^{m-1} \phi_{t_{n+1}}^{-1} f_{u}\left(x_{t_{n}}^{*}, u_{t_{n}}^{*}\right)+D_{t_{m}}^{H}\left(l_{x}\left(x_{t_{m}}^{*}, u_{t_{m}}^{*}\right) \phi_{t_{m}}\right) \sum_{n=0}^{m-1} \phi_{t_{n+1}}^{-1} b_{u}\left(x_{t_{n}}^{*}, u_{t_{n}}^{*}\right)+D_{t_{m}}\left(l_{x}\left(x_{t_{m}}^{*}, u_{t_{m}}^{*}\right) \phi_{t_{m}}\right) \sum_{n=0}^{m-1} \phi_{t_{n+1}}^{-1} \sigma_{u}\left(x_{t_{n}}^{*}, u_{t_{n}}^{*}\right) \\
& \quad+l_{u}\left(x_{t_{m}}^{*}, u_{t_{m}}^{*}\right)+h_{x}\left(x_{t_{N}}^{*}\right) \phi_{t_{N}} \phi_{t_{m+1}}^{-1} f_{u}\left(x_{t_{m}}^{*}, u_{t_{m}}^{*}\right)+D_{t_{N}}^{H}\left(h_{x}\left(x_{t_{N}}^{*}\right) \phi_{t_{N}}\right) \phi_{t_{m+1}}^{-1} b_{u}\left(x_{t_{m}}^{*}, u_{t_{m}}^{*}\right)+D_{t_{N}}\left(h_{x}\left(x_{t_{N}}^{*}\right) \phi_{t_{N}}\right) \phi_{t_{m+1}}^{-1} \sigma_{u}\left(x_{t_{m}}^{*}, u_{t_{m}}^{*}\right)=0 .
\end{aligned}
$$

In order to prove Theorem 1, we begin with the estimation of the first-order variational equation of the state variables.

$$
\sup _{0 \leq k \leq N-1} E\left[\left|x_{t_{k}}^{\varepsilon}-x_{t_{k}}^{*}-y_{t_{k}}^{*}\right|^{2}\right] \leq C \varepsilon^{2} v^{2}
$$

where $y_{t_{k}}^{*}$ is the solution of

Lemma 2. Let the assumptions $(\mathrm{H} 1-\mathrm{H} 3)$ hold. Then,

$$
\left\{\begin{array}{l}
y_{t_{k+1}}^{*}=\left[f_{x}\left(x_{t_{k}}^{*}, u_{t_{k}}^{*}\right) y_{t_{k}}^{*}+\delta_{k m} f_{u}\left(x_{t_{k}}^{*}, u_{t_{k}}^{*}\right) v\right]+\left[b_{x}\left(x_{t_{k}}^{*}, u_{t_{k}}^{*}\right) y_{t_{k}}^{*}+\delta_{k m} b_{u}\left(x_{t_{k}}^{*}, u_{t_{k}}^{*}\right) v\right] g_{t_{k}}+\left[\sigma_{x}\left(x_{t_{k}}^{*}, u_{t_{k}}^{*}\right) y_{t_{k}}^{*}+\delta_{k m} \sigma_{u}\left(x_{t_{k}}^{*}, u_{t_{k}}^{*}\right) v\right] \omega_{t_{k}} \\
y_{t_{0}}^{*}=0
\end{array}\right.
$$

Proof. Let

$$
\begin{aligned}
y_{t_{k+1}}^{\varepsilon}= & x_{t_{k+1}}^{\varepsilon}-x_{t_{k+1}}^{*} \\
= & {\left[f\left(x_{t_{k}}^{\varepsilon}, u_{t_{k}}^{\varepsilon}\right)-f\left(x_{t_{k}}^{*}, u_{t_{k}}^{*}\right)\right]+\left[b\left(x_{t_{k}}^{\varepsilon}, u_{t_{k}}^{\varepsilon}\right)-b\left(x_{t_{k}}^{*}, u_{t_{k}}^{*}\right)\right] g_{t_{k}}+\left[\sigma\left(x_{t_{k}}^{\varepsilon}, u_{t_{k}}^{\varepsilon}\right)-\sigma\left(x_{t_{k}}^{*}, u_{t_{k}}^{*}\right)\right] \omega_{t_{k}} } \\
= & {\left[f\left(x_{t_{k}}^{\varepsilon}, u_{t_{k}}^{\varepsilon}\right)-f\left(x_{t_{k}}^{*}, u_{t_{k}}^{\varepsilon}\right)+f\left(x_{t_{k}}^{*}, u_{t_{k}}^{\varepsilon}\right)-f\left(x_{t_{k}}^{*}, u_{t_{k}}^{*}\right)\right]+\left[b\left(x_{t_{k}}^{\varepsilon}, u_{t_{k}}^{\varepsilon}\right)-b\left(x_{t_{k}}^{*}, u_{t_{k}}^{\varepsilon}\right)+b\left(x_{t_{k}}^{*}, u_{t_{k}}^{\varepsilon}\right)-b\left(x_{t_{k}}^{*}, u_{t_{k}}^{*}\right)\right] g_{t_{k}} } \\
& +\left[\sigma\left(x_{t_{k}}^{\varepsilon}, u_{t_{k}}^{\varepsilon}\right)-\sigma\left(x_{t_{k}}^{*}, u_{t_{k}}^{\varepsilon}\right)+\sigma\left(x_{t_{k}}^{*}, u_{t_{k}}^{\varepsilon}\right)-\sigma\left(x_{t_{k}}^{*}, u_{t_{k}}^{*}\right)\right] \omega_{t_{k}} \\
= & {\left[f_{x}\left(x_{t_{k}}^{*}, u_{t_{k}}^{\varepsilon}\right) y_{t_{k}}^{\varepsilon}+\delta_{k m} f_{u}\left(x_{t_{k}}^{*}, u_{t_{k}}^{*}\right) \varepsilon v\right]+\left[b_{x}\left(x_{t_{k}}^{*}, u_{t_{k}}^{\varepsilon}\right) y_{t_{k}}^{\varepsilon}+\delta_{k m} b_{u}\left(x_{t_{k}}^{*}, u_{t_{k}}^{*}\right) \varepsilon v\right] g_{t_{k}}+\left[\sigma_{x}\left(x_{t_{k}}^{*}, u_{t_{k}}^{\varepsilon}\right) y_{t_{k}}^{\varepsilon}+\delta_{k m} \sigma_{u}\left(x_{t_{k}}^{*}, u_{t_{k}}^{*}\right) \varepsilon v\right] \omega_{t_{k}} . }
\end{aligned}
$$


Under assumption (H2), we have the following moment inequality:

$$
\sup _{0 \leq k \leq N-1} E\left[\left|y_{t_{k}}^{\varepsilon}\right|^{2}\right] \leq C \varepsilon^{2} v^{2}
$$

In fact,

$$
\begin{aligned}
E\left[\left|y_{t_{k+1}}^{\varepsilon}\right|^{2}\right] \leq & 3 E\left\{\left[f_{x}\left(x_{t_{k}}^{*}, u_{t_{k}}^{\varepsilon}\right) y_{t_{k}}^{\varepsilon}+\delta_{k m} f_{u}\left(x_{t_{k}}^{*}, u_{t_{k}}^{*}\right) \varepsilon v\right]^{2}+\left[b_{x}\left(x_{t_{k}}^{*}, u_{t_{k}}^{\varepsilon}\right) y t_{k} \varepsilon+\delta_{k m} b_{u}\left(x_{t_{k}}^{*}, u_{t_{k}}^{*}\right) \varepsilon v\right]^{2}\left|B_{t_{k+1}}-B_{t_{k}}\right|^{2} d+\left[\sigma_{x}\left(x_{t_{k}}^{*}, u_{t_{k}}^{\varepsilon}\right) y_{t_{k}}^{\varepsilon}+\delta_{k m} \sigma_{u}\left(x_{t_{k}}^{*}, u_{t_{k}}^{*}\right) \varepsilon v\right]^{2}\left|t_{k+1}-t_{k}\right| d\right\} \\
= & 3 E\left\{\left[f_{x}^{2}\left(x_{t_{k}}^{*}, u_{t_{k}}^{\varepsilon}\right)+b_{x}^{2}\left(x_{t_{k}}^{*}, u_{t_{k}}^{\varepsilon}\right)\left|t_{k+1}-t_{k}\right|^{2 H} d+\sigma_{x}^{2}\left(x_{t_{k}}^{*}, u_{t_{k}}^{\varepsilon}\right)\left|t_{k+1}-t_{k}\right| d\right]\left(y_{t_{k}}^{\varepsilon}\right)^{2}\right. \\
& +2 \delta_{k m}\left[2 f_{x}\left(x_{t_{k}}^{*}, u_{t_{k}}^{\varepsilon}\right) f_{u}\left(x_{t_{k}}^{*}, u_{t_{k}}^{*}\right)+2 b_{x}\left(x_{t_{k}}^{*}, u_{t_{k}}^{\varepsilon}\right) b_{u}\left(x_{t_{k}}^{*}, u_{t_{k}}^{*}\right)\left|t_{k+1}-t_{k}\right|^{2 H} d+2 \sigma_{x}\left(x_{t_{k}}^{*}, u_{t_{k}}^{\varepsilon}\right) \sigma_{u}\left(x_{t_{k}}^{*}, u_{t_{k}}^{*}\right)\left|t_{k+1}-t_{k}\right| d\right] \varepsilon v y_{t_{k}}^{\varepsilon} \\
& +2 \delta_{k m}\left[f_{u}^{2}\left(x_{t_{k}}^{*}, u_{t_{k}}^{*}\right)+b_{u}^{2}\left(x_{t_{k}}^{*}, u_{t_{k}}^{*}\right)\left|t_{k+1}-t_{k}\right|^{2 H} d+\sigma_{u}^{2}\left(x_{t_{k}}^{*}, u_{t_{k}}^{*}\right)\left|t_{k+1}-t_{k}\right| d\right] \varepsilon^{2} v^{2} \\
\leq & C\left(y_{t_{k}}^{\varepsilon}\right)^{2}+C y_{t_{k}}^{\varepsilon} \varepsilon v+C \varepsilon^{2} v^{2} .
\end{aligned}
$$

We also have $E\left[\left|y_{t_{1}}^{\varepsilon}\right|^{2}\right] \leq C \varepsilon^{2} v^{2}$. So, by repeating this step, moment inequality (25) is verified. Let $y_{t_{k}}^{*}=\lim _{\varepsilon \longrightarrow 0}\left(y_{t_{k}}^{\varepsilon} / \varepsilon\right)$; we have

$$
\left\{\begin{array}{l}
y_{t_{k+1}}^{*}=\left[f_{x}\left(x_{t_{k}}^{*}, u_{t_{k}}^{*}\right) y_{t_{k}}^{*}+\delta_{k m} f_{u}\left(x_{t_{k}}^{*}, u_{t_{k}}^{*}\right) v\right]+\left[b_{x}\left(x_{t_{k}}^{*}, u_{t_{k}}^{*}\right) y_{t_{k}}^{*}+\delta_{k m} b_{u}\left(x_{t_{k}}^{*}, u_{t_{k}}^{*}\right) v\right] g_{t_{k}}+\left[\sigma_{x}\left(x_{t_{k}}^{*}, u_{t_{k}}^{*}\right) y_{t_{k}}^{*}+\delta_{k m} \sigma_{u}\left(x_{t_{k}}^{*}, u_{t_{k}}^{*}\right) v\right] \omega_{t_{k}}, \\
y_{t_{0}}^{*}=0
\end{array}\right.
$$

In order to prove inequality (22), we consider

$$
\begin{aligned}
f\left(x_{t_{k}}^{*}\right. & \left.+y_{t_{k}}^{*}, u_{t_{k}}^{\varepsilon}\right)+b\left(x_{t_{k}}^{*}+y_{t_{k}}^{*}, u_{t_{k}}^{\varepsilon}\right) g_{t_{k}}+\sigma\left(x_{t_{k}}^{*}+y_{t_{k}}^{*}, u_{t_{k}}^{\varepsilon}\right) \omega_{t_{k}} \\
= & {\left[f\left(x_{t_{k}}^{*}, u_{t_{k}}^{\varepsilon}\right)+f_{x}\left(x_{t_{k}}^{*}, u_{t_{k}}^{\varepsilon}\right) y_{t_{k}}^{*}\right]+\left[b\left(x_{t_{k}}^{*}, u_{t_{k}}^{\varepsilon}\right)+b_{x}\left(x_{t_{k}}^{*}, u_{t_{k}}^{\varepsilon}\right) y_{t_{k}}^{*}\right] g_{t_{k}}+\left[\sigma\left(x_{t_{k}}^{*}, u_{t_{k}}^{\varepsilon}\right)+\sigma_{x}\left(x_{t_{k}}^{*}, u_{t_{k}}^{\varepsilon}\right) y_{t_{k}}^{*}\right] \omega_{t_{k}} } \\
= & f\left(x_{t_{k}}^{*}, u_{t_{k}}^{*}\right)+b\left(x_{t_{k}}^{*}, u_{t_{k}}^{*}\right) g_{t_{k}}+\sigma\left(x_{t_{k}}^{*}, u_{t_{k}}^{*}\right) \omega_{t_{k}}+f_{x}\left(x_{t_{k}}^{*}, u_{t_{k}}^{*}\right) y_{t_{k}}^{*}+b_{x}\left(x_{t_{k}}^{*}, u_{t_{k}}^{*}\right) y_{t_{k}}^{*} g_{t_{k}} \\
& +\sigma_{x}\left(x_{t_{k}}^{*}, u_{t_{k}}^{*}\right) y_{t_{k}}^{*} \omega_{t_{k}}+\delta_{k m} f_{u}\left(x_{t_{k}}^{*}, u_{t_{k}}^{*}\right) v+\delta_{k m} b_{u}\left(x_{t_{k}}^{*}, u_{t_{k}}^{*}\right) v g_{t_{k}}+\delta_{k m} \sigma_{u}\left(x_{t_{k}}^{*}, u_{t_{k}}^{*}\right) v \omega_{t_{k}} \\
& +\left[f_{x}\left(x_{t_{k}}^{*}, u_{t_{k}}^{\varepsilon}\right)+f_{x}\left(x_{t_{k}}^{*}, u_{t_{k}}^{*}\right)\right]-\left[b_{x}\left(x_{t_{k}}^{*}, u_{t_{k}}^{\varepsilon}\right)-b_{x}\left(x_{t_{k}}^{*}, u_{t_{k}}^{*}\right)\right] g_{t_{k}}+\left[\sigma_{x}\left(x_{t_{k}}^{*}, u_{t_{k}}^{\varepsilon}\right)-\sigma_{x}\left(x_{t_{k}}^{*}, u_{t_{k}}^{*}\right)\right] \omega_{t_{k}} \\
= & x_{t_{k+1}}^{*}+y_{t_{k+1}}^{*}+\left[f_{x}\left(x_{t_{k}}^{*}, u_{t_{k}}^{\varepsilon}\right)-f_{x}\left(x_{t_{k}}^{*}, u_{t_{k}}^{*}\right)\right]+\left[b_{x}\left(x_{t_{k}}^{*}, u_{t_{k}}^{\varepsilon}\right)-b_{x}\left(x_{t_{k}}^{*}, u_{t_{k}}^{*}\right)\right] g_{t_{k}}+\left[\sigma_{x}\left(x_{t_{k}}^{*}, u_{t_{k}}^{\varepsilon}\right)-\sigma_{x}\left(x_{t_{k}}^{*}, u_{t_{k}}^{*}\right)\right] \omega_{t_{k}} .
\end{aligned}
$$

According to (H2), we have that

$$
\sup _{0 \leq k \leq N-1} E\left[\left|f_{x}\left(x_{t_{k}}^{*}, u_{t_{k}}^{\varepsilon}\right)-f_{x}\left(x_{t_{k}}^{*}, u_{t_{k}}^{*}\right)\right|^{2}+\left|\left(b_{x}\left(x_{t_{k}}^{*}, u_{t_{k}}^{\varepsilon}\right)-b_{x}\left(x_{t_{k}}^{*}, u_{t_{k}}^{*}\right)\right) \omega_{t_{k}}\right|^{2}+\left|\left(\sigma_{x}\left(x_{t_{k}}^{*}, u_{t_{k}}^{\varepsilon}\right)-\sigma_{x}\left(x_{t_{k}}^{*}, u_{t_{k}}^{*}\right)\right) g_{t_{k}}\right|^{2}\right] \leq C \varepsilon^{2} v^{2} \text {. }
$$


Notice that

$$
\begin{aligned}
x_{t_{k+1}}^{*}+y_{t_{k+1}}^{*}= & f\left(x_{t_{k}}^{*}+y_{t_{k}}^{*}, u_{t_{k}}^{\varepsilon}\right)+b\left(x_{t_{k}}^{*}+y_{t_{k}}^{*}, u_{t_{k}}^{\varepsilon}\right) g_{t_{k}}+\sigma\left(x_{t_{k}}^{*}+y_{t_{k}}^{*}, u_{t_{k}}^{\varepsilon}\right) \omega_{t_{k}}-\left[f_{x}\left(x_{t_{k}}^{*}, u_{t_{k}}^{\varepsilon}\right)-f_{x}\left(x_{t_{k}}^{*}, u_{t_{k}}^{*}\right)\right] \\
& -\left[b_{x}\left(x_{t_{k}}^{*}, u_{t_{k}}^{\varepsilon}\right)-b_{x}\left(x_{t_{k}}^{*}, u_{t_{k}}^{*}\right)\right] g_{t_{k}}-\left[\sigma_{x}\left(x_{t_{k}}^{*}, u_{t_{k}}^{\varepsilon}\right)-\sigma_{x}\left(x_{t_{k}}^{*}, u_{t_{k}}^{*}\right)\right] \omega_{t_{k}}, \\
x_{t_{k+1}}^{\varepsilon}= & f\left(x_{t_{k}}^{\varepsilon}, u_{t_{k}}^{\varepsilon}\right)+b\left(x_{t_{k}}^{\varepsilon}, u_{t_{k}}^{\varepsilon}\right) g_{t_{k}}+\sigma\left(x_{t_{k}}^{\varepsilon}, u_{t_{k}}^{\varepsilon}\right) \omega_{t_{k}} .
\end{aligned}
$$

It is easy to obtain

$$
\begin{aligned}
x_{t_{k+1}}^{\varepsilon}-x_{t_{k+1}}^{*}-y_{t_{k+1}}^{*}= & A^{\varepsilon}\left(x_{t_{k}}^{\varepsilon}-x_{t_{k}}^{*}-y_{t_{k}}^{*}\right)+B^{\varepsilon}\left(x_{t_{k}}^{\varepsilon}-x_{t_{k}}^{*}-y_{t_{k}}^{*}\right) \omega_{t_{k}}+C^{\varepsilon}\left(x_{t_{k}}^{\varepsilon}-x_{t_{k}}^{*}-y_{t_{k}}^{*}\right) g_{t_{k}} \\
& +\left[f_{x}\left(x_{t_{k}}^{*}, u_{t_{k}}^{\varepsilon}\right)-f_{x}\left(x_{t_{k}}^{*}, u_{t_{k}}^{*}\right)\right]+\left[b_{x}\left(x_{t_{k}}^{*}, u_{t_{k}}^{\varepsilon}\right)-b_{x}\left(x_{t_{k}}^{*}, u_{t_{k}}^{*}\right)\right] g_{t_{k}}+\left[\sigma_{x}\left(x_{t_{k}}^{*}, u_{t_{k}}^{\varepsilon}\right)-\sigma_{x}\left(x_{t_{k}}^{*}, u_{t_{k}}^{*}\right)\right] \omega_{t_{k}},
\end{aligned}
$$

with

$$
\begin{array}{ll}
A^{\varepsilon}=f_{x}\left(x_{t_{k}}^{\varepsilon}-\theta\left(x_{t_{k}}^{*}+y_{t_{k}}^{*}\right), u_{t_{k}}^{\varepsilon}\right), & \theta \in[0,1], \\
B^{\varepsilon}=b_{x}\left(x_{t_{k}}^{\varepsilon}-\lambda\left(x_{t_{k}}^{*}+y_{t_{k}}^{*}\right), u_{t_{k}}^{\varepsilon}\right), & \lambda \in[0,1], \\
C^{\varepsilon}=\sigma_{x}\left(x_{t_{k}}^{\varepsilon}-\rho\left(x_{t_{k}}^{*}+y_{t_{k}}^{*}\right), u_{t_{k}}^{\varepsilon}\right), & \rho \in[0,1],
\end{array}
$$

which are uniformly bounded according to our assumptions. Through the above derivation, Lemma 2 is proved.
The estimation of the variational equation of the state equation is a critical point to obtain the maximum principle, and we need to solve the above stochastic difference equation.

Lemma 3. There exists a unique bounded solution to the following linear matrix-valued stochastic difference equations:

$$
\left\{\begin{array}{l}
\phi_{t_{k+1}}=\phi_{t_{k}} f_{x}\left(x_{t_{k}}^{*}, u_{t_{k}}^{*}\right)+\phi_{t_{k}} b_{x}\left(x_{t_{k}}^{*}, u_{t_{k}}^{*}\right) g_{t_{k}}+\phi_{t_{k}} \sigma_{x}\left(x_{t_{k}}^{*}, u_{t_{k}}^{*}\right) \omega_{t_{k}}, \\
\phi_{t_{0}}=I_{n \times n} .
\end{array}\right.
$$

Proof. For the uniqueness of the solution to (33), assume that there exists another solution $\phi^{\prime}$. We have

$$
\begin{array}{r}
E\left[\left|\phi_{t_{k}}-\phi_{t_{h}}^{\prime}\right|^{2}\right]=E\left[\left|\phi_{t_{k-1}}-\phi_{t_{k-1}}^{\prime}\right|^{2}\left(f_{x}^{2}\left(x_{t_{k-1}}^{*}, u_{t_{k-1}}^{*}\right)+b_{x}^{2}\left(x_{t_{k-1}}^{*}, u_{t_{k-1}}^{*}\right)\left|t_{k}-t_{k-1}\right|^{2 H} d+\sigma_{x}^{2}\left(x_{t_{k-1}}^{*}, u_{t_{k-1}}^{*}\right)\left|t_{k}-t_{k-1}\right| d\right)\right] \\
\leq C E\left[\left|\phi_{t_{k-1}}-\phi_{t_{k-1}}^{\prime}\right|^{2}\right]
\end{array}
$$

When $k=1$, we have $E\left[\mid \phi_{t_{1}}-\phi_{t_{1}}{ }^{\prime}{ }^{2}\right]=0$. By the inductive method, the uniqueness of the solution to the equations is obtained. For the boundedness of the solution, it is easy to get

$$
\begin{aligned}
E\left[\left|\phi_{t_{k}}\right|^{2}\right]= & E\left[\left|\phi_{t_{k}} \phi_{t_{0}}^{-1}\right|^{2}\right] \\
= & E\left[\left|\phi_{t_{k}} \phi_{t_{k-1}}^{-1} \cdots \phi_{t_{1}} \phi_{t_{0}}^{-1}\right|^{2}\right] \\
= & E\left[\left(f_{x}^{2}\left(x_{t_{k-1}}^{*}, u_{t_{k-1}}^{*}\right)+b_{x}^{2}\left(x_{t_{k-1}}^{*}, u_{t_{k-1}}^{*}\right)\left|t_{k}-t_{k-1}\right|^{2 H} d+\sigma_{x}^{2}\left(x_{t_{k-1}}^{*}, u_{t_{k-1}}^{*}\right)\left|t_{k}-t_{k-1}\right| d\right)\right. \\
& \left.\cdots\left(f_{x}^{2}\left(x_{t_{0}}^{*}, u_{t_{0}}^{*}\right)+b_{x}^{2}\left(x_{t_{0}}^{*}, u_{t_{0}}^{*}\right)\left|t_{1}-t_{0}\right|^{2 H} d+\sigma_{x}^{2}\left(x_{t_{0}}^{*}, u_{t_{0}}^{*}\right)\left|t_{1}-t_{0}\right| d\right)\right]
\end{aligned}
$$

$\leq C$. 
According to Lemma 3, we express $y^{*}$ in an implicit form of $v$ as the following lemma.

Lemma 4. The solution of equations (23) has the following form:

$$
\begin{aligned}
y_{t_{k+1}}^{*}= & \phi_{t_{k+1}} \sum_{n=0}^{k} \phi_{t_{n+1}}^{-1}\left[\delta_{k m} v f_{u}\left(x_{t_{n}}^{*}, u_{t_{n}}^{*}\right)+\delta_{k m} v b_{u}\left(x_{t_{n}}^{*}, u_{t_{n}}^{*}\right) g_{t_{n}}\right. \\
& \left.+\delta_{k m} v \sigma_{u}\left(x_{t_{n}}^{*}, u_{t_{n}}^{*}\right) \omega_{t_{n}}\right] .
\end{aligned}
$$

Proof. According to equations (23) and equations (33), we have

$$
\begin{aligned}
y_{t_{k+1}}^{*}-\phi_{t_{k+1}} \phi_{t_{k}}^{-1} y_{t_{k}}^{*}= & \delta_{k m} v f_{u}\left(x_{t_{k}}^{*}, u_{t_{k}}^{*}\right)+\delta_{k m} v b_{u}\left(x_{t_{k}}^{*}, u_{t_{k}}^{*}\right) g_{t_{k}} \\
& +\delta_{k m} v \sigma_{u}\left(x_{t_{k}}^{*}, u_{t_{k}}^{*}\right) \omega_{t_{k}} .
\end{aligned}
$$

Then, we multiply both sides of the equation by $\phi_{t_{k+1}}^{-1}$, and we obtain

$$
\begin{aligned}
\phi_{t_{k+1}}^{-1} y_{t_{k+1}}^{*}-\phi_{t_{k}}^{-1} y_{t_{k}}^{*}= & \phi_{t_{k+1}}^{-1}\left[\delta_{k m} v f_{u}\left(x_{t_{k}}^{*}, u_{t_{k}}^{*}\right)+\delta_{k m} v b_{u}\left(x_{t_{k}}^{*}, u_{t_{k}}^{*}\right)\right. \\
& \left.\cdot g_{t_{k}} \delta_{k m} v \sigma_{u}\left(x_{t_{k}}^{*}, u_{t_{k}}^{*}\right) \omega_{t_{k}}\right] .
\end{aligned}
$$

By the iterative method, we have

$$
\begin{aligned}
\phi_{t_{k+1}}^{-1} y_{t_{k+1}}^{*}= & \sum_{n=0}^{k} \phi_{t_{n+1}}^{-1}\left[\delta_{k m} v f_{u}\left(x_{t_{n}}^{*}, u_{t_{n}}^{*}\right)+\delta_{k m} v b_{u}\left(x_{t_{n}}^{*}, u_{t_{n}}^{*}\right) g_{t_{n}}\right. \\
& \left.+\delta_{k m} v \sigma_{u}\left(x_{t_{n}}^{*}, u_{t_{n}}^{*}\right) \omega_{t_{n}}\right] .
\end{aligned}
$$

Equation (36) is obtained by multiplying both sides of the equality by $\phi_{t_{k+1}}$.

Lemma 5. We expand the cost functional as

$$
\lim _{\varepsilon \longrightarrow 0} \frac{J\left(u^{\varepsilon}\right)-J\left(u^{*}\right)}{\varepsilon}=E\left[\sum_{k=0}^{N-1}\left(l_{x}\left(x_{t_{k}}^{*}, u_{t_{k}}^{*}\right) y_{t_{k}}^{*}+\delta_{k m} l_{u}\left(x_{t_{k}}^{*}, u_{t_{k}}^{*}\right) v\right)+h_{x}\left(x_{t_{N}}^{*}\right) y_{t_{N}}^{*}\right] \geq 0 .
$$

Proof. Since $\left(x_{t_{k}}^{*}, u_{t_{k}}^{*}\right)$ is optimal, it is natural that

$$
\begin{aligned}
0 & \leq \lim _{\varepsilon \longrightarrow 0} \frac{1}{\mathcal{\varepsilon}}\left\{E\left[\sum_{k=0}^{N-1} l\left(x_{t_{k}}^{\varepsilon}, u_{t_{k}}^{\varepsilon}\right)+h\left(x_{t_{N}}^{\varepsilon}\right)\right]-E\left[\sum_{k=0}^{N-1} l\left(x_{t_{k}}^{*}, u_{t_{k}}^{*}\right)+h\left(x_{t_{N}}^{*}\right)\right]\right\} \\
& =\lim _{\varepsilon} \longrightarrow 0 \frac{1}{\mathcal{E}}\left\{E\left[\sum_{k=0}^{N-1} l\left(x_{t_{k}}^{*}+y_{t_{k}}^{\varepsilon}, u_{t_{k}}^{\varepsilon}\right)+h\left(x_{t_{N}}^{*}+y_{t_{N}}^{\varepsilon}\right)\right]-E\left[\sum_{k=0}^{N-1} l\left(x_{t_{k}}^{*}, u_{t_{k}}^{*}\right)+h\left(x_{t_{N}}^{*}\right)\right]\right\} \\
& =\lim _{\varepsilon} \frac{1}{\mathcal{E}}\left\{E\left[\sum_{k=0}^{N-1} l\left(x_{t_{k}}^{*}+y_{t_{k}}^{\varepsilon}, u_{t_{k}}^{*}\right)+h\left(x_{t_{N}}^{*}+y_{t_{N}}^{\varepsilon}\right)\right]-E\left[\sum_{k=0}^{N-1} l\left(x_{t_{k}}^{*}, u_{t_{k}}^{*}\right)+h\left(x_{t_{N}}^{*}\right)\right]\right. \\
& \left.+E\left[\sum_{k=0}^{N-1} l\left(x_{t_{k}}^{*}+y_{t_{k}}^{\varepsilon}, u_{t_{k}}^{\varepsilon}\right)-l\left(x_{t_{k}}^{*}+y_{t_{k}}^{\varepsilon}, u_{t_{k}}^{*}\right)\right]\right\} .
\end{aligned}
$$

By Lemma 2, we have

$$
\begin{aligned}
0 & \leq \lim _{\varepsilon \longrightarrow 0} \frac{1}{\varepsilon}\left\{E\left[\sum_{k=0}^{N-1}\left(l_{x}\left(x_{t_{k}}^{*}, u_{t_{k}}^{*}\right) y_{t_{k}}^{\varepsilon}+\delta_{k m} l_{u}\left(x_{t_{k}}^{*}, u_{t_{k}}^{*}\right) \varepsilon v\right)\right]+E\left[\sum_{k=0}^{N-1}\left(l_{x}\left(x_{t_{k}}^{*}, u_{t_{k}}^{\varepsilon}\right)-l_{x}\left(x_{t_{k}}^{*}, u_{t_{k}}^{*}\right)\right) y_{t_{k}}^{\varepsilon}\right]+E\left[h_{x}\left(x_{t_{N}}^{*}\right) y_{t_{N}}^{\varepsilon}\right]\right\} \\
& =E\left[\sum_{k=0}^{N-1}\left(l_{x}\left(x_{t_{k}}^{*}, u_{t_{k}}^{*}\right) y_{t_{k}}^{*}+\delta_{k m} l_{u}\left(x_{t_{k}}^{*}, u_{t_{k}}^{*}\right) v\right)+h_{x}\left(x_{t_{N}}^{*}\right) y_{t_{N}}^{*}\right] .
\end{aligned}
$$

Then, variational inequality (40) is derived. 
According to equation (36), we substitute the solution of (23) in the functional $J(u)$.

$$
\begin{aligned}
\lim _{\varepsilon \longrightarrow 0} \frac{J\left(u^{\varepsilon}\right)-J\left(u^{*}\right)}{\varepsilon}= & E\left[\sum_{k=0}^{N-1}\left(l_{x}\left(x_{t_{k}}^{*}, u_{t_{k}}^{*}\right) y_{t_{k}}^{*}+\delta_{k m} l_{u}\left(x_{t_{k}}^{*}, u_{t_{k}}^{*}\right) v\right)+h_{x}\left(x_{t_{N}}^{*}\right) y_{t_{N}}^{*}\right] \\
= & E\left[\sum_{k=0}^{N-1} l_{x}\left(x_{t_{k}}^{*}, u_{t_{k}}^{*}\right) \phi_{t_{k}} \sum_{n=0}^{k-1} \phi_{t_{n+1}}^{-1} \delta_{k m} v f_{u}\left(x_{t_{n}}^{*}, u_{t_{n}}^{*}\right)\right]+E\left[\sum_{k=0}^{N-1} l_{x}\left(x_{t_{k}}^{*}, u_{t_{k}}^{*}\right) \phi_{t_{k}} \sum_{n=0}^{k-1} \phi_{t_{n+1}}^{-1} \delta_{k m} v b_{u}\left(x_{t_{n}}^{*}, u_{t_{n}}^{*}\right) g_{t_{n}}\right] \\
& +E\left[\sum_{k=0}^{N-1} l_{x}\left(x_{t_{k}}^{*}, u_{t_{k}}^{*}\right) \phi_{t_{k}} \sum_{n=0}^{k-1} \phi_{t_{n+1}}^{-1} \delta_{k m} v \sigma_{u}\left(x_{t_{n}}^{*}, u_{t_{n}}^{*}\right) \omega_{t_{n}}\right]+E\left[\sum_{k=0}^{N-1} \delta_{k m} l_{u}\left(x_{t_{k}}^{*}, u_{t_{k}}^{*}\right) v\right] \\
& +E\left[h_{x}\left(x_{t_{N}}^{*}\right) \phi_{t_{N}} \sum_{n=0}^{N-1} \phi_{t_{n+1}}^{-1} \delta_{k m} v f_{u}\left(x_{t_{n}}^{*}, u_{t_{n}}^{*}\right)\right] \\
& +E\left[h_{x}\left(x_{t_{N}}^{*}\right) \phi_{t_{N}} \sum_{n=0}^{N-1} \phi_{t_{n+1}}^{-1} \delta_{k m} v b_{u}\left(x_{t_{n}}^{*}, u_{t_{n}}^{*}\right) g_{t_{n}}\right]+E\left[h_{x}\left(x_{t_{N}}^{*}\right) \phi_{t_{N}} \sum_{n=0}^{N-1} \phi_{t_{n+1}}^{-1} \delta_{k m} v \sigma_{u}\left(x_{t_{n}}^{*}, u_{t_{n}}^{*}\right) \omega_{t_{n}}\right] .
\end{aligned}
$$

Lemma 6. To deal with the terms with fractional noise, we have the following duality formula of Malliavin calculus:

$$
\begin{aligned}
E\left[F_{t_{i}} \int_{0}^{t_{i}} z(t) \mathrm{d} B_{t}^{H}\right] & =E\left[F_{t_{i}} \sum_{k=0}^{i-1} \int_{t_{k}}^{t_{k+1}} z_{t_{k}} 1_{\left[t_{k}, t_{k+1}\right.}(t) \mathrm{d} B_{t}^{H}\right] \\
& =E\left[F_{t_{i}} \sum_{k=0}^{i-1} z_{t_{k}}\left(B_{t_{k+1}}^{H}-B_{t_{k}}^{H}\right)\right] \\
& =E\left[F_{t_{i}} \sum_{k=0}^{i-1} z_{t_{k}} g_{t_{k}}\right] .
\end{aligned}
$$$$
E\left[F_{t_{i}} \sum_{k=0}^{i-1} z_{t_{k}} g_{t_{k}}\right]=E\left[D_{t_{i}}^{H} F_{t_{i}} \sum_{k=0}^{i-1} z_{t_{k}}\right] \text {. }
$$

Proof. By Lemma 1, we have

$$
E\left[F_{t_{i}} \int_{0}^{t_{i}} z(t) \mathrm{d} B_{t}^{H}\right]=E\left[\int_{0}^{t_{i}}\left(D_{t}^{H} F_{t_{i}}\right) z(t) \mathrm{d} t\right] .
$$

Let $z(t)=\sum_{k=0}^{N-1} z_{t_{k}} 1_{\left[t_{k}, t_{k+1}\right)}(t)$; then, the left hand of (45) The right hand of equality (45) is is derived as

$$
\begin{aligned}
E\left[\int_{0}^{t_{i}}\left(D_{t}^{H} F_{t_{i}}\right) z(t) \mathrm{d} t\right] & =E\left[\sum_{k=0}^{i-1} \int_{t_{k}}^{t_{k+1}}\left(D_{t} F_{t_{i}}(t)\right) \sum_{k=0}^{N-1} z_{t_{k}} 1_{\left[t_{k}, t_{k+1}\right)}(t) \mathrm{d} t\right] \\
& =E\left[\sum_{k=0}^{i-1}\left(z_{t_{k}} \int_{t_{k}}^{t_{k+1}} D_{t} F_{t_{i}} \mathrm{~d} t\right)\right] \\
& =E\left[D_{t_{i}} F_{t_{i}} \sum_{k=0}^{i-1} z_{t_{k}}\right] .
\end{aligned}
$$

This completes the proof of Lemma 6 . 
By Lemma 6, we rewrite equality (43) as

$$
\begin{aligned}
\lim _{\varepsilon \longrightarrow 0} \frac{J\left(u^{\varepsilon}\right)-J\left(u^{*}\right)}{\varepsilon}= & E\left[\sum_{k=0}^{N-1} l_{x}\left(x_{t_{k}}^{*}, u_{t_{k}}^{*}\right) \phi_{t_{k}} \sum_{n=0}^{k-1} \phi_{t_{n+1}}^{-1} \delta_{k m} f_{u}\left(x_{t_{n}}^{*}, u_{t_{n}}^{*}\right) v\right]+E\left[\sum_{k=0}^{N-1} D_{t_{k}}^{H}\left(l_{x}\left(x_{t_{k}}^{*}, u_{t_{k}}^{*}\right) \phi_{t_{k}}\right) \sum_{n=0}^{k-1} \phi_{t_{n+1}}^{-1} \delta_{k m} v b_{u}\left(x_{t_{n}}^{*}, u_{t_{n}}^{*}\right)\right] \\
& +E\left[\sum_{k=0}^{N-1} D_{t_{k}}\left(l_{x}\left(x_{t_{k}}^{*}, u_{t_{k}}^{*}\right) \phi_{t_{k}}\right) \sum_{n=0}^{k-1} \phi_{t_{n+1}}^{-1} \delta_{k m} v \sigma_{u}\left(x_{t_{n}}^{*}, u_{t_{n}}^{*}\right)\right]+E\left[\sum_{k=0}^{N-1} \delta_{k m} l_{u}\left(x_{t_{k}}^{*}, u_{t_{k}}^{*}\right) v\right] \\
& +E\left[\sum_{k=0}^{N-1} \phi_{t_{k+1}^{-1}}^{-1} f_{u}\left(x_{t_{k}}^{*}, u_{t_{k}}^{*}\right) h_{x}\left(x_{t_{N}}^{*}\right) \phi_{t_{N}} \delta_{k m} v\right] \\
& +E\left[\sum_{k=0}^{N-1} \phi_{t_{k+1}^{-1}} b_{u}\left(x_{t_{k}}^{*}, u_{t_{k}}^{*}\right) D_{t_{N}}^{H}\left(h_{x}\left(x_{t_{N}}^{*}\right) \phi_{t_{N}}\right) \delta_{k m} v\right]+E\left[\sum_{k=0}^{N-1} \phi_{t_{k+1}}^{-1} \sigma_{u}\left(x_{t_{k}}^{*}, u_{t_{k}}^{*}\right) D_{t_{N}}\left(h_{x}\left(x_{t_{N}}^{*}\right) \phi_{t_{N}}\right) \delta_{k m} v\right] .
\end{aligned}
$$

For arbitrary $v$ that is not equal to zero, we have

$$
\lim _{\varepsilon \longrightarrow 0} \frac{J\left(u^{\varepsilon}\right)-J\left(u^{*}\right)}{\varepsilon} \geq 0 .
$$

Then, we obtain the following general maximum principle:

$$
\begin{aligned}
& l_{x}\left(x_{t_{m}}^{*}, u_{t_{m}}^{*}\right) \phi_{t_{m}} \sum_{n=0}^{m-1} \phi_{t_{n+1}}^{-1} f_{u}\left(x_{t_{n}}^{*}, u_{t_{n}}^{*}\right)+D_{t_{m}}^{H}\left(l_{x}\left(x_{t_{m}}^{*}, u_{t_{m}}^{*}\right) \phi_{t_{m}}\right) \sum_{n=0}^{m-1} \phi_{t_{n+1}}^{-1} b_{u}\left(x_{t_{n}}^{*}, u_{t_{n}}^{*}\right)+D_{t_{m}}\left(l_{x}\left(x_{t_{m}}^{*}, u_{t_{m}}^{*}\right) \phi_{t_{m}}\right) \sum_{n=0}^{m-1} \phi_{t_{n+1}}^{-1} \sigma_{u}\left(x_{t_{n}}^{*}, u_{t_{n}}^{*}\right) \\
& \quad+l_{u}\left(x_{t_{m}}^{*}, u_{t_{m}}^{*}\right)+h_{x}\left(x_{t_{N}}^{*}\right) \phi_{t_{N}} \phi_{t_{m+1}}^{-1} f_{u}\left(x_{t_{m}}^{*}, u_{t_{m}}^{*}\right)+D_{t_{N}}^{H}\left(h_{x}\left(x_{t_{N}}^{*}\right) \phi_{t_{N}}\right) \phi_{t_{m+1}}^{-1} b_{u}\left(x_{t_{m}}^{*}, u_{t_{m}}^{*}\right)+D_{t_{N}}\left(h_{x}\left(x_{t_{N}}^{*}\right) \phi_{t_{N}}\right) \phi_{t_{m+1}}^{-1} \sigma_{u}\left(x_{t_{m}}^{*}, u_{t_{m}}^{*}\right)=0 .
\end{aligned}
$$

This completes Theorem 1.

\section{Applications to the Linear- Quadratic Problem}

In this section, we apply Theorem 1 to a stochastic linearquadratic optimal control problem.

Consider the controlled system as follows:

$$
\left\{\begin{array}{l}
x_{t_{k+1}}=A_{t_{k}} x_{t_{k}}+B_{t_{k}} v_{t_{k}} g_{t_{k}}+C_{t_{k}} v_{t_{k}} \omega_{t_{k}}, \quad k=0,1, \ldots, N-1, \\
x_{t_{0}}=x_{0}
\end{array}\right.
$$

with the cost functional

$$
J(v)=E\left[\sum_{k=0}^{N-1}\left(x_{t_{k}}^{\top} M_{t_{k}} x_{t_{k}}+v_{t_{k}}^{\top} Q_{t_{k}} v_{t_{k}}\right)+x_{t_{N}}^{\top} M_{t_{N}} x_{t_{N}}\right] \text {. }
$$

Here, $A_{t_{k}} \in \mathbb{R}^{n}$ and $B_{t_{k}} \in \mathbb{R}^{n}$ and, $M_{t_{k}}, k=1,2, \ldots, N$ and $Q_{t_{k}}, k=1,2, \ldots, N-1$, are positive matrices. According to (23), the variational equation of the state equations is

$$
\left\{\begin{array}{l}
y_{t_{k+1}}^{*}=A_{t_{k}} y_{t_{k}}^{*}+B_{t_{k}} \delta_{k m} v g_{t_{k}}+C_{t_{k}} \delta_{k m} v \omega_{t_{k}} \\
y_{t_{0}}^{*}=0
\end{array}\right.
$$

In this case, we get $\phi_{t_{k}}=\prod_{i=0}^{k-1} A_{t_{i}}$. We express $y^{*}$ in an implicit form of $v$ as

$$
y_{t_{k}}^{*}=\phi_{t_{k}} \sum_{n=0}^{k-1} \phi_{t_{n+1}}^{-1}\left(B_{t_{n}} \delta_{k m} v g_{t_{n}}+C_{t_{n}} \delta_{k m} v \omega_{t_{n}}\right) .
$$

Applying Theorem 1, we obtain the maximum principle as follows:

$$
\begin{aligned}
& D_{t_{m}}^{H}\left(2 x_{t_{m}}^{*} M_{t_{m}} \phi_{t_{m}}\right) \sum_{n=0}^{m-1} \phi_{t_{n+1}}^{-1} B_{t_{n}}+D_{t_{m}}\left(2 x_{t_{m}}^{*} M_{t_{m}} \phi_{t_{m}}\right) \sum_{n=0}^{m-1} \phi_{t_{n+1}}^{-1} C_{t_{n}} \\
& \quad+2 u_{t_{m}}^{*} Q_{t_{m}}+D_{t_{N}}^{H}\left(2 x_{t_{N}}^{*} M_{t_{N}} \phi_{t_{N}}\right) \phi_{t_{m+1}}^{-1} B_{t_{m}} \\
& +D_{t_{N}}\left(2 x_{t_{N}}^{*} M_{t_{N}} \phi_{t_{N}}\right) \phi_{t_{m+1}}^{-1} C_{t_{m}}=0 .
\end{aligned}
$$

In this special case, we get the optimal control directly as 


$$
\begin{aligned}
u_{t_{m}}^{*}= & -\frac{1}{2 Q_{t_{m}}}\left[D_{t_{m}}^{H}\left(2 x_{t_{m}}^{*} M_{t_{m}} \phi_{t_{m}}\right) \sum_{n=0}^{m-1} \phi_{t_{n+1}}^{-1} B_{t_{n}}+D_{t_{m}}\left(2 x_{t_{m}}^{*} M_{t_{m}} \phi_{t_{m}}\right) \sum_{n=0}^{m-1} \phi_{t_{n+1}}^{-1} C_{t_{n}}+D_{t_{N}}^{H}\left(2 x_{t_{N}}^{*} M_{t_{N}} \phi_{t_{N}}\right) \phi_{t_{m+1}}^{-1} B_{t_{m}}\right. \\
& \left.+D_{t_{N}}\left(2 x_{t_{N}}^{*} M_{t_{N}} \phi_{t_{N}}\right) \phi_{t_{m+1}}^{-1} C_{t_{m}}\right] .
\end{aligned}
$$

\section{Conclusion}

In this paper, the necessary optimality conditions of the discrete stochastic optimal control problems driven by both fractional noise and white noise are derived. The admissible control region is not necessarily convex. The stochastic variational inequalities are obtained by applying the classical variation method and the iterative method. The Malliavin calculus is used to derive the maximum principle for our problems. In fact, we obtain a more general maximum principle. We also apply our maximum principle to a discrete linear-quadratic optimal control problem, and the optimal control is obtained.

\section{Data Availability}

No data were used to support this study.

\section{Conflicts of Interest}

The authors declare that they have no conflicts of interest.

\section{Acknowledgments}

This research was partially supported by NSFC (grant 11871244), the Special Funds of Provincial Industrial Innovation of Jilin Province, China (no. 2017C028-1), Project of Science and Technology Development of Jilin Province, China (no. 20190201302JC), and China Automobile Industry Innovation and Development Joint Fund (U1564213).

\section{References}

[1] L. S. Pontryagin, V. G. Boltyanski, R. V. Gamkrelidze, and E. F. Mischenko, Mathematical Theory of Optimal Processes, Wiley, New York, NY, USA, 1962.

[2] J. M. Holtzman and H. Halkin, "Directional convexity and the maximum principle for discrete systems," SIAM Journal on Control, vol. 4, no. 2, pp. 213-275, 1966.

[3] H.-Q. Müller, "Maximum principles for discrete-time nonlinear stochastic control systems," Optimization, vol. 27, no. 4, pp. 343-354, 1993.

[4] M. J. Mardanov, S. T. Malik, and N. I. Mahmudov, "On the theory of necessary optimality conditions in discrete systems," Advances in Difference Equations, vol. 2015, no. 1, 15 pages, 2015.

[5] H. J. Kushner, "Necessary conditions for continuous parameter stochastic optimization problems," SIAM Journal on Control, vol. 10, no. 3, pp. 550-565, 1972.

[6] L. W. Neustadt, "An abstract variational theory with applications to a broad class of optimization problems. I. General theory," SIAM Journal on Control, vol. 4, no. 3, pp. 505-527, 1966.
[7] U. G. Haussmann, "A discrete approximation to optimal stochastic controls," Analysis \& Optimization of Stochastic Systems, pp. 229-241, 1978.

[8] J.-M. Bismut, "An introductory approach to duality in optimal stochastic control," SIAM Review, vol. 20, no. 1, pp. 62-78, 1978.

[9] S. Peng, "A general stochastic maximum principle for optimal control problems," SIAM Journal on Control and Optimization, vol. 28, no. 4, pp. 966-979, 1990.

[10] Y. Han, Y. Hu, and J. Song, "Maximum principle for general controlled systems driven by fractional Brownian motions," Applied Mathematics \& Optimization, vol. 67, no. 2, pp. 279-322, 2013.

[11] C. A. Burdet and S. P. Sethi, "On the maximum principle for a class of discrete dynamical systems with lags," Journal of Optimization Theory and Applications, vol. 19, no. 3, pp. 445-454, 1976.

[12] X. Lin and W. Zhang, "A maximum principle for optimal control of discrete-time stochastic systems with multiplicative noise," IEEE Transactions on Automatic Control, vol. 60, no. 4, pp. 1121-1126, 2015.

[13] Y. Hu, "Integral transformations and anticipative calculus for fractional Brownian motions," Memoirs of the American Mathematical Society, vol. 175, no. 825, 2005. 